\title{
Oscillatory properties of half-linear difference equations: two-term perturbations
}

\section{Simona Fišnarová*}

\section{"Correspondence:}

fisnarov@mendelu.cz

Department of Mathematics,

Mendel University in Brno,

Zemědělská 1, Brno, CZ-613 00,

Czech Republic

\begin{abstract}
We consider the nonoscillatory half-linear difference equation

$$
\Delta\left(r_{k} \Phi\left(\Delta x_{k}\right)\right)+c_{k} \Phi\left(x_{k+1}\right)=0, \quad \Phi(x):=|x|^{p-1} \operatorname{sgn} x, p>1,
$$

and we study the influence of the perturbations $\tilde{r}, \tilde{c}$ on the oscillatory properties of the equation

$$
\Delta\left[\left(r_{k}+\tilde{r}_{k}\right) \Phi\left(\Delta x_{k}\right)\right]+\left(c_{k}+\tilde{c}_{k}\right) \Phi\left(x_{k+1}\right)=0 .
$$

The presented oscillation and nonoscillation criteria are obtained using the variational principle and the so-called modified Riccati technique.
\end{abstract}

\section{Introduction}

In this article, we study oscillatory properties of the second-order half-linear difference equation of the form

$$
L\left[x_{k}\right]:=\Delta\left(r_{k} \Phi\left(\Delta x_{k}\right)\right)+c_{k} \Phi\left(x_{k+1}\right)=0, \quad \Phi(x):=|x|^{p-1} \operatorname{sgn} x, p>1,
$$

where $r, c$ are real-valued sequences, $r_{k} \neq 0$. If $p=2$, then (1) reduces to the linear SturmLiouville difference equation

$$
\Delta\left(r_{k} \Delta x_{k}\right)+c_{k} x_{k+1}=0 .
$$

The basic qualitative theory of (1) has been established in the article [1] and is summarized in the books [2, 3]. Many oscillatory properties of (1) are very similar to that of (2), however the absence of the linearity requires sometimes to use different methods in half-linear case.

In this article, we deal with the so-called perturbation principle. We suppose that equation (1) is nonoscillatory and that $h$ is a solution of (1) and we give conditions under which the perturbed equation

$$
\tilde{L}\left[x_{k}\right]:=\Delta\left[\left(r_{k}+\tilde{r}_{k}\right) \Phi\left(\Delta x_{k}\right)\right]+\left(c_{k}+\tilde{c}_{k}\right) \Phi\left(x_{k+1}\right)=0,
$$

where $r_{k}+\tilde{r}_{k} \neq 0$, is oscillatory or nonoscillatory. Similar problem has been studied in [4, 5], where the case $\tilde{r}_{k}=0$ has been considered. We extend some results of those papers to

(c) 2012 Fišnarová; licensee Springer. This is an Open Access article distributed under the terms of the Creative Commons Attribution License (http://creativecommons.org/licenses/by/2.0), which permits unrestricted use, distribution, and reproduction in any medium, provided the original work is properly cited. 
the general case $\tilde{r}_{k} \neq 0$ and we also show that the assumption $\lim _{k \rightarrow \infty} r_{k} h_{k} \Phi\left(\Delta h_{k}\right)=\infty$ considered in [4] can be replaced by alternative conditions. We are motivated also by the results of [6,7], where the two-term perturbations of the half-linear differential equation

$$
\left(r(t) \Phi\left(x^{\prime}\right)\right)^{\prime}+c(t) \Phi(x)=0, \quad r(t)>0
$$

are studied.

The article is organized as follows. In the next section, we recall the basic methods of oscillation theory for (1), in particular the variational principle and the Riccati technique. Section 3 is devoted to the so-called modified Riccati technique. In Section 4, we present the main results of this article, the oscillation and nonoscillation criteria for the perturbed equation (3) and in the last section, we show how the results can be applied to the perturbed equation of the Euler type.

\section{Preliminaries}

Oscillatory properties of (1) are defined using the concept of the generalized zero. We say that a solution $x$ of (1) has a generalized zero in an interval $(m, m+1]$ if $x_{m} \neq 0$ and $r_{m} x_{m} x_{m+1} \leq 0$. Equation (1) is said to be disconjugate on an interval $[m, n]$ if any solution of (1) has at most one generalized zero on $(m, n+1]$ and the solution for which $x_{m}=0$ has no generalized zero on $(m, n+1]$. Consequently, equation (1) is said to be nonoscillatory if there exists $m \in \mathbb{N}$ such that this equation is disconjugate on $[m, n]$ for every $n>m$. In the opposite case, (1) is said to be oscillatory.

One of the basic methods used to investigate (non)oscillation of (1) is the variational technique which relates nonoscillation of (1) to a positivity of a certain $p$-degree functional.

Lemma 1 [1] Equation (1) is nonoscillatory if and only if there exists $m \in \mathbb{N}$ such that

$$
\mathcal{F}(y, m, \infty):=\sum_{k=m}^{\infty}\left[r_{k}\left|\Delta y_{k}\right|^{p}-c_{k}\left|y_{k+1}\right|^{p}\right]>0
$$

for every nontrivial sequence $y \in U(m)$, where

$$
U(m):=\left\{y=\left\{y_{k}\right\}_{k=1}^{\infty} ; y_{k}=0, k \leq m, \exists n>m: y_{k}=0, k \geq n\right\} .
$$

The second basic method used is the Riccati technique which is based on the relationship between nonoscillation of (1) and the solvability (in a neighborhood of infinity) of the Riccati-type equation

$$
R\left[w_{k}\right]:=w_{k+1}+c_{k}-\frac{r_{k} w_{k}}{\Phi\left(\Phi^{-1}\left(r_{k}\right)+\Phi^{-1}\left(w_{k}\right)\right)}=0,
$$

where $\Phi^{-1}$ is the inverse function of $\Phi$, i.e. $\Phi^{-1}(s)=|s|^{q-1} \operatorname{sgn} s, q:=\frac{p}{p-1}$. Indeed, if $x$ is a solution of (1) such that $x_{k} \neq 0$ on some discrete interval $[m, \infty)$, then $w_{k}=r_{k} \Phi\left(\Delta x_{k} / x_{k}\right)$ is a solution of $(4)$ on $[m, \infty)$. More precisely, we have the following equivalent statements.

Lemma 2 [1] The following statements are equivalent: 
(i) Equation (1) is nonoscillatory.

(ii) There exists a solution $w$ of (4) such that $r_{k}+w_{k}>0$ for large $k$.

(iii) There exists a solution $w$ of the Riccati inequality $R\left[w_{k}\right] \leq 0$ such that $r_{k}+w_{k}>0$ for large $k$.

If (1) is nonoscillatory, then there exists a solution $w_{k}$ of (4) such that $r_{k}+w_{k}>0$ for large $k$. Among all solutions $w$ with this property, there is the so-called minimal solution $\tilde{w}$ for which $\tilde{w}_{k}<w_{k}$ on some interval $[m, \infty)$, where $r_{k}+w_{k}>0$ and $r_{k}+\tilde{w}_{k}>0$. The minimal solution $\tilde{w}$ can be constructed as follows. Let (1) be disconjugate on $[n, \infty)$ and let $N>n$. Denote by $x^{N}$ the solution of (1) which satisfies $x_{N}^{N}=0, x_{N+1}^{N} \neq 0$ and let $w^{N}=r \Phi\left(\Delta x^{N} / x^{N}\right)$ be the solution of (4) associated with $x^{N}$. Then

$$
\tilde{w}_{k}=\lim _{N \rightarrow \infty} w_{k}^{N} \quad \text { for every } k \in[n+1, \infty)
$$

For details of this construction see [5]. The solution $\tilde{x}$ of (1) which is associated with the minimal solution $\tilde{w}$ of (4) by the substitution $\tilde{w}=r \Phi(\Delta \tilde{x} / \tilde{x})$, is called the recessive solution of $(1)$. The recessive solution is defined uniquely up to the multiplication by a real constant.

Next, we formulate a comparison statement for minimal solutions of two Riccati equations. The Riccati equation associated with (3) is

$$
\tilde{R}\left[w_{k}\right]:=w_{k+1}+c_{k}+\tilde{c}_{k}-\frac{\left(r_{k}+\tilde{r}_{k}\right) w_{k}}{\Phi\left(\Phi^{-1}\left(r_{k}+\tilde{r}_{k}\right)+\Phi^{-1}\left(w_{k}\right)\right)}=0 .
$$

\section{Lemma 3 [5]}

(i) Let (3) be nonoscillatory and let $\tilde{r}_{k} \leq 0, \tilde{c}_{k} \geq 0$ for large $k$. Further, let $\tilde{w}$ and $\bar{w}$ be the minimal solutions of the corresponding Riccati equations (4) and (6), respectively.

Then there exists $m \in \mathbb{Z}$ such that $\tilde{w}_{k} \leq \bar{w}_{k}$ for $k \in[m, \infty)$.

(ii) If $c_{k} \geq 0$ and $\sum^{\infty} r_{k}^{1-q}=\infty$, then the minimal solution of (4) satisfies $\tilde{w}_{k} \geq 0$ for large $k$.

Note that the statement (ii) of the previous lemma is a special case of the statement (i). Condition $\sum^{\infty} r_{k}^{1-q}=\infty$ implies that the recessive solution of the equation $\Delta\left(r_{k} \Phi\left(\Delta x_{k}\right)\right)=$ 0 is a constant sequence, hence the minimal solution of the associated Riccati equation is $w=0$ and this is compared with the minimal solution of (4).

\section{Modified Riccati equation and related results}

In this section, we suppose that equation (1) is nonoscillatory, by $h$ we denote a positive solution of this equation and suppose that both the coefficients $r_{k}, r_{k}+\tilde{r}_{k}$ are positive for large $k$. This sign restriction is needed when proving inequalities (10), (11) and estimate (12) below, for details see [4]. Since these estimates play the crucial role in the (non)oscillation criteria based on the modified Riccati technique presented in this section, we suppose that $r_{k}>0, r_{k}+\tilde{r}_{k}>0$ for large $k$ throughout the whole Section 3 .

Denote

$$
G_{k}:=\left(r_{k}+\tilde{r}_{k}\right) h_{k} \Phi\left(\Delta h_{k}\right),
$$


define the function

$$
H(k, v):=v+\left(r_{k}+\tilde{r}_{k}\right) h_{k+1} \Phi\left(\Delta h_{k}\right)-\frac{\left(r_{k}+\tilde{r}_{k}\right)\left(v+G_{k}\right) h_{k+1}^{p}}{\Phi\left(h_{k}^{q} \Phi^{-1}\left(r_{k}+\tilde{r}_{k}\right)+\Phi^{-1}\left(v+G_{k}\right)\right)},
$$

and consider the so-called modified Riccati equation

$$
R_{m}\left[v_{k}\right]:=\Delta v_{k}+\left[\Delta\left(\tilde{r}_{k} \Phi\left(\Delta h_{k}\right)\right)+\tilde{c}_{k} \Phi\left(h_{k+1}\right)\right] h_{k+1}+H\left(k, v_{k}\right)=0
$$

We show that there is a relation between operators given in (6) and (9) and estimate the function $H(k, v)$ by a term involving $v^{2}$, which, in turn, enables us to compare Riccati equation associated with (3) with the Riccati equation related to a certain linear equation.

Lemma 4 Let $w$ be a sequence such that $r_{k}+\tilde{r}_{k}+w_{k} \neq 0$ and suppose that $v_{k}=h_{k}^{p} w_{k}-G_{k}$. Then

$$
R_{m}\left[v_{k}\right]=h_{k+1}^{p} \tilde{R}\left[w_{k}\right]
$$

Proof By a direct computation

$$
\Delta v_{k}=h_{k+1}^{p} w_{k+1}-h_{k}^{p} w_{k}-\Delta\left(\left(r_{k}+\tilde{r}_{k}\right) \Phi\left(\Delta h_{k}\right)\right) h_{k+1}-\left(r_{k}+\tilde{r}_{k}\right)\left|\Delta h_{k}\right|^{p}
$$

and

$$
\begin{aligned}
H\left(k, v_{k}\right) & =h_{k}^{p} w_{k}+\left(r_{k}+\tilde{r}_{k}\right)\left|\Delta h_{k}\right|^{p}-\frac{\left(r_{k}+\tilde{r}_{k}\right) h_{k}^{p} h_{k+1}^{p} w_{k}}{\Phi\left(h_{k}^{q} \Phi^{-1}\left(r_{k}+\tilde{r}_{k}\right)+\Phi^{-1}\left(h_{k}^{p} w_{k}\right)\right)} \\
& =h_{k}^{p} w_{k}+\left(r_{k}+\tilde{r}_{k}\right)\left|\Delta h_{k}\right|^{p}-\frac{\left(r_{k}+\tilde{r}_{k}\right) h_{k+1}^{p} w_{k}}{\Phi\left(\Phi^{-1}\left(r_{k}+\tilde{r}_{k}\right)+\Phi^{-1}\left(w_{k}\right)\right)} .
\end{aligned}
$$

Hence,

$$
\Delta v_{k}+\Delta\left(\left(r_{k}+\tilde{r}_{k}\right) \Phi\left(\Delta h_{k}\right)\right) h_{k+1}+H\left(k, v_{k}\right)=h_{k+1}^{p} w_{k+1}-\frac{\left(r_{k}+\tilde{r}_{k}\right) h_{k+1}^{p} w_{k}}{\Phi\left(\Phi^{-1}\left(r_{k}+\tilde{r}_{k}\right)+\Phi^{-1}\left(w_{k}\right)\right)} .
$$

Adding the term $h_{k+1}^{p}\left(c_{k}+\tilde{c}_{k}\right)$ to both sides of the last equality, we obtain

$$
\Delta v_{k}+h_{k+1} \tilde{L}\left[h_{k}\right]+H\left(k, v_{k}\right)=h_{k+1}^{p} \tilde{R}\left[w_{k}\right],
$$

where $\tilde{L}$ is the operator defined in (3). Since $h$ is a solution of (1), we have the required identity.

Lemma 5 Let $H(k, v)$ be defined in (8).

(i) It holds $H(k, v) \geq 0$ for $v>-\left(r_{k}+\tilde{r}_{k}\right) h_{k}\left(\Phi\left(h_{k}\right)+\Phi\left(\Delta h_{k}\right)\right)$ with the equality if and only if $v=0$.

(ii) Suppose that $h_{k} \Delta h_{k}>0$ for $k \in[m, \infty)$ and denote $R_{k}=\frac{2}{q}\left(r_{k}+\tilde{r}_{k}\right) h_{k} h_{k+1}\left|\Delta h_{k}\right|^{p-2}$. Then we have the following inequalities for $v \geq 0$ and $k \in[m, \infty)$ :

$$
\begin{aligned}
& \left(R_{k}+v\right) H(k, v) \geq v^{2}, \quad p \in(1,2], \\
& \left(R_{k}+v\right) H(k, v) \leq v^{2}, \quad p \geq 2 .
\end{aligned}
$$


(iii) Suppose that $\liminf _{k \rightarrow \infty} G_{k}>0$, then for large k:

$$
\left(R_{k}+v\right) H(k, v)=v^{2}(1+o(1)) \quad \text { as } v \rightarrow 0 .
$$

(iv) Suppose that $h_{k} \Delta h_{k}>0$ for large $k$ and

$$
\begin{array}{ll}
\sum^{\infty} \frac{\Delta h_{k}}{h_{k}}=\infty, & \sum^{\infty}\left(\frac{\Delta h_{k}}{h_{k}}\right)^{2}<\infty, \\
0<\liminf _{k \rightarrow \infty} G_{k}, & \limsup _{k \rightarrow \infty} G_{k}<\infty .
\end{array}
$$

Then $\sum^{\infty} H(k, v)=\infty$ for every $v>0$.

Proof The proof of the statements (i), (ii), (iii) (with $r_{k}+\tilde{r}_{k}$ replaced by $r_{k}$ ) can be found in [4].

(iv) Let $v>0$ be arbitrary. The function $H(k, v)$ can be written as follows:

$$
\begin{aligned}
H(k, v) & =v+\left(r_{k}+\tilde{r}_{k}\right) h_{k+1} \Phi\left(\Delta h_{k}\right)-\frac{\left(r_{k}+\tilde{r}_{k}\right)\left(v+G_{k}\right) h_{k+1}^{p}}{\Phi\left(h_{k}^{q} \Phi^{-1}\left(r_{k}+\tilde{r}_{k}\right)+\Phi^{-1}\left(v+G_{k}\right)\right)} \\
& =v+\frac{h_{k+1}}{h_{k}} G_{k}-\frac{\left(v+G_{k}\right) h_{k+1}^{p}}{\Phi\left(h_{k}^{q}+\Phi^{-1}\left(\frac{v+G_{k}}{r_{k}+\tilde{r}_{k}}\right)\right)} \\
& =v+\frac{h_{k+1}}{h_{k}} G_{k}-\left(\frac{h_{k+1}}{h_{k}}\right)^{p} \frac{\left(v+G_{k}\right)}{\Phi\left(1+\Phi^{-1}\left(\frac{v+G_{k}}{\left(r_{k}+\tilde{r}_{k}\right) h_{k}^{p}}\right)\right)} .
\end{aligned}
$$

The second condition in (13) implies $\frac{\Delta h_{k}}{h_{k}} \rightarrow 0$ as $k \rightarrow \infty$, hence, using the formula

$$
(1+x)^{s}=\sum_{j=0}^{\infty}\left(\begin{array}{l}
s \\
j
\end{array}\right) x^{j}=1+s x+O\left(x^{2}\right), \quad \text { as } x \rightarrow 0, s \in \mathbb{R}
$$

we have

$$
\left(\frac{h_{k+1}}{h_{k}}\right)^{p}=\left(1+\frac{\Delta h_{k}}{h_{k}}\right)^{p}=1+p \frac{\Delta h_{k}}{h_{k}}+O\left(\left(\frac{\Delta h_{k}}{h_{k}}\right)^{2}\right) \text { as } k \rightarrow \infty
$$

and since the first condition in (14) holds, we have

$$
\begin{aligned}
\Phi^{-1}\left(\frac{v+G_{k}}{\left(r_{k}+\tilde{r}_{k}\right) h_{k}^{p}}\right) & =\Phi^{-1}\left(\frac{G_{k}\left(1+\frac{v}{G_{k}}\right)}{\left(r_{k}+\tilde{r}_{k}\right) h_{k}^{p}}\right) \\
& =\frac{\Delta h_{k}}{h_{k}} \Phi^{-1}\left(1+\frac{v}{G_{k}}\right) \rightarrow 0 \text { as } k \rightarrow \infty .
\end{aligned}
$$

Consequently, again using (15) and conditions (14),

$$
\begin{aligned}
{\left[\Phi\left(1+\Phi^{-1}\left(\frac{v+G_{k}}{\left(r_{k}+\tilde{r}_{k}\right) h_{k}^{p}}\right)\right)\right]^{-1} } & =\left(1+\frac{\Delta h_{k}}{h_{k}} \Phi^{-1}\left(1+\frac{v}{G_{k}}\right)\right)^{1-p} \\
& =1+(1-p) \frac{\Delta h_{k}}{h_{k}} \Phi^{-1}\left(1+\frac{v}{G_{k}}\right)+O\left(\left(\frac{\Delta h_{k}}{h_{k}}\right)^{2}\right),
\end{aligned}
$$


as $k \rightarrow \infty$. Hence, $H(k, v)$ can be written in the form

$$
\begin{aligned}
H(k, v)= & v+\left(1+\frac{\Delta h_{k}}{h_{k}}\right) G_{k}-\left(1+p \frac{\Delta h_{k}}{h_{k}}+O\left(\left(\frac{\Delta h_{k}}{h_{k}}\right)^{2}\right)\right)\left(v+G_{k}\right) \\
& \times\left(1-(p-1) \frac{\Delta h_{k}}{h_{k}} \Phi^{-1}\left(1+\frac{v}{G_{k}}\right)+O\left(\left(\frac{\Delta h_{k}}{h_{k}}\right)^{2}\right)\right) \\
= & v+G_{k}+\frac{\Delta h_{k}}{h_{k}} G_{k} \\
& -\left(v+G_{k}\right)\left[1-(p-1) \frac{\Delta h_{k}}{h_{k}} \Phi^{-1}\left(1+\frac{v}{G_{k}}\right)+p \frac{\Delta h_{k}}{h_{k}}+O\left(\left(\frac{\Delta h_{k}}{h_{k}}\right)^{2}\right)\right] \\
= & \frac{\Delta h_{k}}{h_{k}} G_{k}+G_{k}\left(1+\frac{v}{G_{k}}\right)\left[(p-1) \frac{\Delta h_{k}}{h_{k}} \Phi^{-1}\left(1+\frac{v}{G_{k}}\right)-p \frac{\Delta h_{k}}{h_{k}}\right]+O\left(\left(\frac{\Delta h_{k}}{h_{k}}\right)^{2}\right) \\
= & \frac{\Delta h_{k}}{h_{k}} G_{k}\left[1+(p-1)\left|1+\frac{v}{G_{k}}\right|^{q}-p\left(1+\frac{v}{G_{k}}\right)\right]+O\left(\left(\frac{\Delta h_{k}}{h_{k}}\right)^{2}\right),
\end{aligned}
$$

as $k \rightarrow \infty$.

Consider the function $A(x):=1+(p-1)|1+x|^{q}-p(1+x)$. By a direct computation $A^{\prime}(x)=$ $p\left(\Phi^{-1}(1+x)-1\right)$ and $A^{\prime \prime}(x)=q|1+x|^{q-2}$. This means that the function $A(x)$ has a local minimum at $\tilde{x}=0$ and it is positive and increasing for $x>0$.

By conditions (14) there exist constants $c>0, d>0$ such that $c<G_{k}<d$ for large $k$. Since $\frac{v}{G_{k}}>\frac{v}{d}$, we have

$$
1+(p-1)\left|1+\frac{v}{G_{k}}\right|^{q}-p\left(1+\frac{v}{G_{k}}\right)>1+(p-1)\left|1+\frac{v}{d}\right|^{q}-p\left(1+\frac{v}{d}\right)=A\left(\frac{v}{d}\right)>0 .
$$

Consequently,

$$
H(k, v)>c A\left(\frac{v}{d}\right) \frac{\Delta h_{k}}{h_{k}}+O\left(\left(\frac{\Delta h_{k}}{h_{k}}\right)^{2}\right) .
$$

The convergence of the series $\sum^{\infty} O\left(\left(\frac{\Delta h_{k}}{h_{k}}\right)^{2}\right)$ follows from the second condition in (13). This means, by the first condition in (13), that $\sum^{\infty} H(k, v)=\infty$.

\section{Oscillation and nonoscillation criteria}

We start with a statement based on the variational principle. This statement generalizes a result of [5] dealing with the case $\tilde{r}_{k}=0$. Here, we do not need the sign restriction on $r_{k}$, $r_{k}+\tilde{r}_{k}$, we suppose that $r_{k} \neq 0$ and $r_{k}+\tilde{r}_{k} \neq 0$ for large $k$.

Theorem 1 Let $h$ be the recessive solution of (1) and let $\Delta\left(\tilde{r}_{k} / r_{k}\right) \leq 0$ for large $k$. If

$$
\sum^{\infty}\left[\Delta\left(\tilde{r}_{k} \Phi\left(\Delta h_{k}\right)\right)+\tilde{c}_{k} \Phi\left(h_{k+1}\right)\right] h_{k+1}=\infty
$$


Proof The proof is based on Lemma 1. Let $N_{0} \in \mathbb{N}$ be arbitrary and let $K, L, M, N$ be positive integers satisfying $N_{0}<K<L<M<N$, where $K$ is such that (1) is disconjugate on $[K, \infty)$ and $\Delta\left(\tilde{r}_{k} / r_{k}\right) \leq 0$ holds on this interval, and $L$ is such that $h$ has no generalized zero on $[L, \infty)$, i.e. $r_{k} h_{k} h_{k+1}>0$ on $[L, \infty)$. The values $M, N$ will be specified later. Define the sequence

$$
y_{k}:= \begin{cases}0, & k=N_{0}, \ldots, K, \\ f_{k}, & k=K, \ldots, L, \\ h_{k}, & k=L, \ldots, M, \\ g_{k}, & k=M, \ldots, N, \\ 0, & k \geq N,\end{cases}
$$

where $f$ is any sequence satisfying $f_{K}=0, f_{L}=h_{L}$ and $g$ is a solution of (1) for which $g_{M}=h_{M}$, $g_{N}=0$. The fact that such a solution really exists follows from the disconjugacy of (1) on $[K, \infty)$ and from the homogeneity of the solution space of (1). Indeed, if $x$ is a solution of (1) given by $x_{N}=0, x_{N-1} \neq 0$, then $r_{k} x_{k} x_{k+1}>0$ on $[M, N-2]$ and $g_{k}=\frac{h_{M}}{x_{M}} x_{k}$ is the solution of (1) satisfying the required boundary conditions. It also holds $r_{k} g_{k} g_{k+1}>0$ on $[M, N-2]$.

Denote

$$
w^{[h]}:=r \frac{\Phi(\Delta h)}{\Phi(h)}, \quad w^{[g]}:=r \frac{\Phi(\Delta g)}{\Phi(g)}
$$

the corresponding solutions of Riccati equation (4). Set

$$
\mathcal{F}(y ; K, L-1)=\sum_{k=K}^{L-1}\left[\left(r_{k}+\tilde{r}_{k}\right)\left|\Delta f_{k}\right|^{p}-\left(c_{k}+\tilde{c}_{k}\right)\left|f_{k+1}\right|^{p}\right]=: \alpha_{1} \in \mathbb{R} .
$$

Next, using summation by parts, and since $h$ is a solution of (1), we have

$$
\begin{aligned}
\mathcal{F}(y ; L, M-1)= & \sum_{k=L}^{M-1}\left[\left(r_{k}+\tilde{r}_{k}\right)\left|\Delta h_{k}\right|^{p}-\left(c_{k}+\tilde{c}_{k}\right)\left|h_{k+1}\right|^{p}\right] \\
= & \left.\left(r_{k}+\tilde{r}_{k}\right) \Phi\left(\Delta h_{k}\right) h_{k}\right|_{L} ^{M} \\
& -\sum_{k=L}^{M-1}\left[\Delta\left[\left(r_{k}+\tilde{r}_{k}\right) \Phi\left(\Delta h_{k}\right)\right]+\left(c_{k}+\tilde{c}_{k}\right) \Phi\left(h_{k+1}\right)\right] h_{k+1} \\
= & \left.\left(1+\frac{\tilde{r}_{k}}{r_{k}}\right) w_{k}^{[h]}\left|h_{k}\right|^{p}\right|_{L} ^{M}-\sum_{k=L}^{M-1}\left[\Delta\left(\tilde{r}_{k} \Phi\left(\Delta h_{k}\right)\right)+\tilde{c}_{k} \Phi\left(h_{k+1}\right)\right] h_{k+1} \\
= & \left(1+\frac{\tilde{r}_{M}}{r_{M}}\right) w_{M}^{[h]}\left|h_{M}\right|^{p}-\sum_{k=L}^{M-1}\left[\Delta\left(\tilde{r}_{k} \Phi\left(\Delta h_{k}\right)\right)+\tilde{c}_{k} \Phi\left(h_{k+1}\right)\right] h_{k+1}+\alpha_{2},
\end{aligned}
$$

where $\alpha_{2} \in \mathbb{R}$.

Concerning interval $[M, N]$, since $g_{N}=0$ and $h$ has no generalized zero in this interval, it follows from [5, Lemma 3] that $w_{k}^{[g]}<w_{k}^{[h]}$ for $k \in[M, N-1]$. This means that

$$
r_{k} \Phi\left(\Delta g_{k}\right) g_{k}<r_{k} \frac{\Phi\left(\Delta h_{k}\right)}{\Phi\left(h_{k}\right)}\left|g_{k}\right|^{p}, \quad k \in[M, N-1] .
$$


At the same time, $0<\frac{r_{k} g_{k} g_{k+1}}{r_{k} h_{k} h_{k+1}}=\frac{g_{k}}{h_{k}} \frac{g_{k+1}}{h_{k+1}}$ for $k \in[M, N-2]$, hence the condition $g_{M}=h_{M}$ implies $g_{k} h_{k}>0$ for $k \in[M, N-1]$. Hence, (20) implies $r_{k}\left(\Delta g_{k} h_{k}-\Delta h_{k} g_{k}\right)<0$ for $k \in$ $[M, N-1]$ and

$$
\Delta\left(\frac{g_{k}}{h_{k}}\right)=\frac{r_{k}\left(\Delta g_{k} h_{k}-g_{k} \Delta h_{k}\right)}{r_{k} h_{k} h_{k+1}}<0, \quad k \in[M, N-1] .
$$

Next, again summing by parts and using the boundary conditions and the fact that $g$ is a solution of (1),

$$
\begin{aligned}
\mathcal{F}(y ; M, N-1) & =\sum_{k=M}^{N-1}\left[\left(r_{k}+\tilde{r}_{k}\right)\left|\Delta g_{k}\right|^{p}-\left(c_{k}+\tilde{c}_{k}\right)\left|g_{k+1}\right|^{p}\right] \\
& =-\left(1+\frac{\tilde{r}_{M}}{r_{M}}\right) w_{M}^{[g]}\left|h_{M}\right|^{p}-\sum_{k=M}^{N-1}\left[\Delta\left(\tilde{r}_{k} \Phi\left(\Delta g_{k}\right)\right)+\tilde{c}_{k} \Phi\left(g_{k+1}\right)\right] g_{k+1} .
\end{aligned}
$$

Using (20) and since $\Delta\left(\tilde{r}_{k} / r_{k}\right)<0$,

$$
\begin{aligned}
\sum_{k=M}^{N-1} & {\left[\Delta\left(\tilde{r}_{k} \Phi\left(\Delta g_{k}\right)\right)+\tilde{c}_{k} \Phi\left(g_{k+1}\right)\right] g_{k+1} } \\
& =\sum_{k=M}^{N-1}\left[\Delta\left(\frac{\tilde{r}_{k}}{r_{k}} r_{k} \Phi\left(\Delta g_{k}\right)\right) g_{k+1}+\tilde{c}_{k}\left|g_{k+1}\right|^{p}\right] \\
& =\sum_{k=M}^{N-1}\left[\Delta\left(\frac{\tilde{r}_{k}}{r_{k}}\right) r_{k+1} \Phi\left(\Delta g_{k+1}\right) g_{k+1}+\frac{\tilde{r}_{k}}{r_{k}} \Delta\left(r_{k} \Phi\left(\Delta g_{k}\right)\right) g_{k+1}+\tilde{c}_{k}\left|g_{k+1}\right|^{p}\right] \\
& \geq \sum_{k=M}^{N-1}\left[\Delta\left(\frac{\tilde{r}_{k}}{r_{k}}\right) r_{k+1} \frac{\Phi\left(\Delta h_{k+1}\right)}{\Phi\left(h_{k+1}\right)}\left|g_{k+1}\right|^{p}-\frac{\tilde{r}_{k}}{r_{k}} c_{k}\left|g_{k+1}\right|^{p}+\tilde{c}_{k}\left|g_{k+1}\right|^{p}\right] \\
= & \sum_{k=M}^{N-1}\left[\Delta\left(\frac{\tilde{r}_{k}}{r_{k}}\right) r_{k+1} \Phi\left(\Delta h_{k+1}\right) h_{k+1}-\frac{\tilde{r}_{k}}{r_{k}} c_{k}\left|h_{k+1}\right|^{p}+\tilde{c}_{k}\left|h_{k+1}\right|^{p}\right] \frac{\left|g_{k+1}\right|^{p}}{\left|h_{k+1}\right|^{p}} \\
= & \sum_{k=M}^{N-1}\left[\Delta\left(\frac{\tilde{r}_{k}}{r_{k}}\right) r_{k+1} \Phi\left(\Delta h_{k+1}\right) h_{k+1}+\frac{\tilde{r}_{k}}{r_{k}} \Delta\left(r_{k} \Phi\left(\Delta h_{k}\right)\right) h_{k+1}+\tilde{c}_{k}\left|h_{k+1}\right|^{p}\right] \frac{\left|g_{k+1}\right|^{p}}{\left|h_{k+1}\right|^{p}} \\
& =\sum_{k=M}^{N-1}\left[\Delta\left(\tilde{r}_{k} \Phi\left(\Delta h_{k}\right)\right)+\tilde{c}_{k} \Phi\left(h_{k+1}\right)\right] h_{k+1} \frac{\left|g_{k+1}\right|^{p}}{\left|h_{k+1}\right|^{p}} .
\end{aligned}
$$

Since (21) holds, by the second mean value theorem of summation calculus, see [8, Lemma 3.2], there exists $n \in[M, N-1]$ such that

$$
\begin{gathered}
\sum_{k=M}^{N-1}\left[\Delta\left(\tilde{r}_{k} \Phi\left(\Delta h_{k}\right)\right)+\tilde{c}_{k} \Phi\left(h_{k+1}\right)\right] h_{k+1} \frac{\left|g_{k+1}\right|^{p}}{\left|h_{k+1}\right|^{p}} \\
\geq \frac{\left|g_{M}\right|^{p}}{\left|h_{M}\right|^{p}} \sum_{k=M}^{n-1}\left[\Delta\left(\tilde{r}_{k} \Phi\left(\Delta h_{k}\right)\right)+\tilde{c}_{k} \Phi\left(h_{k+1}\right)\right] h_{k+1} \\
\quad+\frac{\left|g_{N}\right|^{p}}{\left|h_{N}\right|^{p}} \sum_{k=n}^{N-1}\left[\Delta\left(\tilde{r}_{k} \Phi\left(\Delta h_{k}\right)\right)+\tilde{c}_{k} \Phi\left(h_{k+1}\right)\right] h_{k+1}
\end{gathered}
$$




$$
=\sum_{k=M}^{n-1}\left[\Delta\left(\tilde{r}_{k} \Phi\left(\Delta h_{k}\right)\right)+\tilde{c}_{k} \Phi\left(h_{k+1}\right)\right] h_{k+1} .
$$

Combining the above computations, we have

$$
\mathcal{F}(y ; M, N-1) \leq-\left(1+\frac{\tilde{r}_{M}}{r_{M}}\right) w_{M}^{[g]}\left|h_{M}\right|^{p}-\sum_{k=M}^{n-1}\left[\Delta\left(\tilde{r}_{k} \Phi\left(\Delta h_{k}\right)\right)+\tilde{c}_{k} \Phi\left(h_{k+1}\right)\right] h_{k+1} .
$$

Consequently, using (18), (19) and (22), we obtain

$$
\begin{aligned}
\mathcal{F}\left(y ; N_{0}, \infty\right)= & \sum_{k=N_{0}}^{\infty}\left[\left(r_{k}+\tilde{r}_{k}\right)\left|\Delta y_{k}\right|^{p}-\left(c_{k}+\tilde{c}_{k}\right)\left|y_{k+1}\right|^{p}\right] \\
= & \mathcal{F}(y ; K, L-1)+\mathcal{F}(y ; L, M-1)+\mathcal{F}(y ; M, N-1) \\
\leq & \alpha_{1}+\alpha_{2}+\left(1+\frac{\tilde{r}_{M}}{r_{M}}\right)\left|h_{M}\right|^{p}\left(w_{M}^{[h]}-w_{M}^{[g]}\right) \\
& -\sum_{k=L}^{n-1}\left[\Delta\left(\tilde{r}_{k} \Phi\left(\Delta h_{k}\right)\right)+\tilde{c}_{k} \Phi\left(h_{k+1}\right)\right] h_{k+1} .
\end{aligned}
$$

Let $\alpha_{3}>0$ be arbitrary. It follows from condition (16) that $M$ can be taken so large that

$$
\sum_{k=L}^{n-1}\left[\Delta\left(\tilde{r}_{k} \Phi\left(\Delta h_{k}\right)\right)+\tilde{c}_{k} \Phi\left(h_{k+1}\right)\right] h_{k+1} \geq \alpha_{1}+\alpha_{2}+\alpha_{3} .
$$

Since $h$ is the recessive solution of (1), i.e. $w^{[h]}$ is the minimal solution of (4), from its construction (5) we have

$$
w_{M}^{[h]}=\lim _{N \rightarrow \infty} w_{M}^{[g]}
$$

Hence, $N$ can be taken so large that

$$
\left(1+\frac{\tilde{r}_{M}}{r_{M}}\right)\left|h_{M}\right|^{p}\left(w_{M}^{[h]}-w_{M}^{[g]}\right)<\alpha_{3} .
$$

Consequently, if $M, N$ are taken as above, then

$$
\mathcal{F}\left(y ; N_{0}, \infty\right) \leq 0,
$$

and this means that equation (3) is oscillatory by Lemma 1 . The proof is complete.

The following results are based on the modified Riccati technique. Here we suppose that $h$ is a positive solution of the nonoscillatory equation (1) and $r_{k}>0, r_{k}+\tilde{r}_{k}>0$ for large k. Denote

$$
C_{k}:=\left[\Delta\left(\tilde{r}_{k} \Phi\left(\Delta h_{k}\right)\right)+\tilde{c}_{k} \Phi\left(h_{k+1}\right)\right] h_{k+1}, \quad R_{k}:=\frac{2}{q}\left(r_{k}+\tilde{r}_{k}\right) h_{k} h_{k+1}\left|\Delta h_{k}\right|^{p-2}
$$


First we present a statement, where, using the global inequalities (10), (11), equation (3) is compared with the linear equation

$$
\Delta\left(R_{k} \Delta y_{k}\right)+C_{k} y_{k+1}=0
$$

This statement generalizes [4, Theorems 3.1 and 3.2].

Theorem 2 Let $h$ be a solution of (1) such that $h_{k}>0, h_{k} \Delta h_{k}>0$ for large $k$.

(i) Let $p \geq 2$, and suppose that $C_{k} \geq 0$ for large $k$ and $\sum^{\infty} \frac{1}{R_{k}}=\infty$. If the linear equation (24) is nonoscillatory, then equation (3) is also nonoscillatory.

(ii) Let $p \leq 2$, and suppose that $h$ is the recessive solution of (1), $\tilde{r}_{k} \leq 0, \tilde{c}_{k} \geq 0$ for large $k$. If the linear equation (24) is oscillatory, then equation (3) is also oscillatory.

Proof (i) Nonoscillation of equation (24) means that there exists a solution $v$ of the Riccati equation

$$
\Delta v_{k}+C_{k}+\frac{v_{k}^{2}}{R_{k}+v_{k}}=0
$$

such that $R_{k}+v_{k}>0$ for large $k$. Assumptions of the theorem imply, in view of Lemma 3(ii), that $v_{k} \geq 0$ for large $k$. Hence, from Lemma 5(ii) it follows

$$
\left(R_{k}+v_{k}\right) H\left(k, v_{k}\right) \leq v_{k}^{2} \quad \text { for large } k
$$

i.e. $v_{k}$ solves the inequality

$$
\Delta v_{k}+C_{k}+H\left(k, v_{k}\right) \leq 0
$$

for large $k$. Consequently, by Lemma 4, we have that the sequence $w=h^{-p}(v+G)$ is a solution of Riccati inequality $\tilde{R}\left[w_{k}\right] \leq 0$. Moreover,

$$
r_{k}+\tilde{r}_{k}+w_{k}=r_{k}+\tilde{r}_{k}+h_{k}^{-p} v_{k}+\left(r_{k}+\tilde{r}_{k}\right) \Phi\left(\Delta h_{k} / h_{k}\right)=h_{k}^{-p} v_{k}+\left(1+\frac{\tilde{r}_{k}}{r_{k}}\right)\left(r_{k}+w_{k}^{[h]}\right),
$$

where $w_{k}^{[h]}$ is a solution of (4) related to a nonoscillatory solution $h$ of (1) and hence $r_{k}+$ $w_{k}^{[h]}>0$ for large $k$. Since $v_{k} \geq 0$ for large $k$, we have $r_{k}+\tilde{r}_{k}+\tilde{w}_{k}>0$ for large $k$. Hence, equation (3) is nonoscillatory according to Lemma 2.

(ii) Suppose, by contradiction, that equation (3) is nonoscillatory and let $w$ be a solution of the associated Riccati equation (6). Then, by Lemma 4,v $=h^{p} w-G$ solves the modified Riccati equation (9). Since $\tilde{r}_{k} \leq 0, \tilde{c}_{k} \geq 0$, it follows from Lemma 3 that $w_{k} \geq w_{k}^{[h]}$ for large $k$, where $w_{k}^{[h]}=r_{k} \Phi\left(\Delta h_{k} / h_{k}\right)$ is the minimal solution of (4). This means that $v_{k}=h_{k}^{p} w_{k}$ $-G_{k}=h_{k}^{p}\left(w_{k}-\left(1+\frac{\tilde{r}_{k}}{r_{k}}\right) w_{k}^{[h]}\right) \geq 0$ for large $k$. Applying inequality (10) we have

$$
\left(R_{k}+v_{k}\right) H\left(k, v_{k}\right) \geq v_{k}^{2}
$$

for large $k$ and hence $v$ solves the inequality

$$
\Delta v_{k}+C_{k}+\frac{v_{k}^{2}}{R_{k}+v_{k}} \leq 0
$$


which, together with the fact that $R_{k}+v_{k}>0$ for large $k$, means that (1) is nonoscillatory

The next two statements are based on the estimate (12) which holds for all $p>1$. Hence we do not have to distinguish between the cases $p>2, p<2$. The idea of the proofs is the same as in Theorem 2 and we skip the proofs since they are similar to that in [4, Theorems 4.1 and 4.2]. The only difference is that we replace $r_{k}$ by $r_{k}+\tilde{r}_{k}$.

Theorem 3 Suppose that $h$ is a solution of (1) such that $h_{k}>0, h_{k} \Delta h_{k}>0$ for large $k$ and let

$$
\sum^{\infty} \frac{1}{R_{k}}=\infty, \quad \sum^{\infty} C_{k}<\infty, \quad C_{k} \geq 0 \text { for large } k
$$

and

$$
\liminf _{k \rightarrow \infty} G_{k}>0
$$

If there exists $\varepsilon>0$ such that the linear equation

$$
\Delta\left(R_{k} \Delta y_{k}\right)+(1+\varepsilon) C_{k} y_{k+1}=0
$$

is nonoscillatory, then equation (3) is also nonoscillatory.

Theorem 4 Suppose that $h$ is the recessive solution of (1) such that $h_{k}>0, h_{k} \Delta h_{k}>0$, $\tilde{c}_{k} \geq 0, \tilde{r}_{k} \leq 0$ for large $k$, conditions (25) hold and

$$
\lim _{k \rightarrow \infty} G_{k}=\infty
$$

If there exists $\varepsilon>0$ such that the linear equation

$$
\Delta\left(R_{k} \Delta y_{k}\right)+(1-\varepsilon) C_{k} y_{k+1}=0
$$

is oscillatory, then equation (3) is also oscillatory.

The next statement is a version of Theorem 4. In the proof of the statement we use Lemma 5(iv). This enables to replace condition (28) by alternative conditions. This statement is new also in case $\tilde{r}=0$.

Theorem 5 Suppose that $h$ is the recessive solution of (1) such that $h_{k}>0, h_{k} \Delta h_{k}>0$, $\tilde{c}_{k} \geq 0, \tilde{r}_{k} \leq 0$ for large $k$ and conditions (25), (13) and (14) hold. If there exists $\varepsilon>0$ such that the linear equation (29) is oscillatory, then equation (3) is also oscillatory.

Proof Suppose, by contradiction, that equation (3) is nonoscillatory and let $w$ be a solution of the associated Riccati equation (6) and $v=h^{p} w-G$ the associated solution of the modified Riccati equation (9). Similarly as in the proof of Theorem 2(ii), we conclude that $v_{k} \geq 0$ for large $k$. Since $C_{k} \geq 0$ for large $k$ and $H\left(k, v_{k}\right)$ is nonnegative, we have for large $k$

$$
\Delta v_{k}=-C_{k}-H\left(k, v_{k}\right) \leq 0 .
$$


This means that there exists a finite $\operatorname{limit}_{\lim _{k \rightarrow \infty}} v_{k}$. Summing the modified Riccati equation from $N$ to $k, N$ being sufficiently large, and using the fact that $v$ is nonnegative, we obtain

$$
\nu_{N} \geq \sum_{j=N}^{k} C_{j}+\sum_{j=N}^{k} H\left(j, v_{j}\right) .
$$

Letting $k \rightarrow \infty$ and since $\sum^{\infty} C_{k}<\infty$, we have $\sum^{\infty} H\left(k, v_{k}\right)<\infty$. From Lemma 5(iv) it follows that $\lim _{k \rightarrow \infty} v_{k}=0$. Now we can use the estimate (12). There exists $N_{1}$ such that

$$
H\left(k, v_{k}\right)>\left(1-\frac{\varepsilon}{2}\right) \frac{v_{k}^{2}}{R_{k}+v_{k}} \quad \text { for } k>N_{1} .
$$

We have

$$
R_{k}=\frac{2}{q}\left(r_{k}+\tilde{r}_{k}\right) h_{k} h_{k+1}\left|\Delta h_{k}\right|^{p-2}=\frac{2}{q} G_{k}\left(1+\frac{h_{k}}{\Delta h_{k}}\right)>\frac{2}{q} G_{k} .
$$

Hence, by (14), $\lim _{k \rightarrow \infty} \frac{v_{k}}{R_{k}}=0$ and similarly as in [4, Theorem 4.1], we obtain for sufficiently large $k$ the estimate $\frac{1}{\frac{R_{k}}{1-\varepsilon}+v_{k}}<\frac{1-\frac{\varepsilon}{2}}{R_{k}+v_{k}}$. Hence,

$$
H\left(k, v_{k}\right)>\left(1-\frac{\varepsilon}{2}\right) \frac{v_{k}^{2}}{R_{k}+v_{k}}>\frac{v_{k}^{2}}{\frac{R_{k}}{1-\varepsilon}+v_{k}}
$$

for sufficiently large $k$. This means that $v_{k}$ solves the inequality

$$
\Delta v_{k}+C_{k}+\frac{v_{k}^{2}}{\frac{R_{k}}{1-\varepsilon}+v_{k}}<0
$$

which is the Riccati inequality associated with equation (29) and since $\frac{R_{k}}{1-\varepsilon}+v_{k}>0$, we have nonoscillation of (29). This is a contradiction.

It is known (see [9]), that if $c_{k} \geq 0, r_{k}>0, \sum^{\infty} r_{k}^{-1}=\infty, \sum^{\infty} c_{k}<\infty$, then the linear equation (2) is nonoscillatory provided

$$
\limsup _{k \rightarrow \infty} \sum^{k-1} r_{j}^{-1} \sum_{j=k}^{\infty} c_{j}<\frac{1}{4},
$$

and it is oscillatory provided

$$
\liminf _{k \rightarrow \infty} \sum^{k-1} r_{j}^{-1} \sum_{j=k}^{\infty} c_{j}>\frac{1}{4} .
$$

Applying this criterion to equations (27), (29), we obtain the following result.

Corollary 1 Suppose that $h$ is a solution of (1) such that $h_{k}>0, h_{k} \Delta h_{k}>0$ for large $k$ and let conditions (25) and (26) be satisfied. 
(i) If

$$
\limsup _{k \rightarrow \infty} \sum^{k-1} \frac{1}{\left(r_{j}+\tilde{r}_{j}\right) h_{j} h_{j+1}\left|\Delta h_{j}\right|^{p-2}} \sum_{j=k}^{\infty} C_{j}<\frac{1}{2 q},
$$

then equation (3) is nonoscillatory.

(ii) Let moreover $h$ be the recessive solution of (1), $\tilde{c}_{k} \geq 0, \tilde{r}_{k} \leq 0$ for large $k$ and let either condition (28) or conditions (13) and (14) be satisfied. If

$$
\liminf _{k \rightarrow \infty} \sum^{k-1} \frac{1}{\left(r_{j}+\tilde{r}_{j}\right) h_{j} h_{j+1}\left|\Delta h_{j}\right|^{p-2}} \sum_{j=k}^{\infty} C_{j}>\frac{1}{2 q}
$$

then equation (3) is oscillatory.

Proof Consider, e.g., the case (i), the case (ii) is analogous. Condition (32) can be written in the form

$$
\limsup _{k \rightarrow \infty} \sum^{k-1} R_{j}^{-1} \sum_{j=k}^{\infty} C_{j}<\frac{1}{4} .
$$

Consequently, there exists $\varepsilon>0$ such that

$$
\limsup _{k \rightarrow \infty} \sum^{k-1} R_{j}^{-1} \sum_{j=k}^{\infty}(1+\varepsilon) C_{j}<\frac{1}{4},
$$

hence equation (27) is nonoscillatory and this implies, by Theorem 3, nonoscillation of (3).

Remark 1 If $\tilde{r}_{k}=0, \tilde{c}_{k} \geq 0$, then the statements of Theorem 2 reduce to [4, Theorems 3.1 and 3.2]. More precisely, (non)oscillation of

$$
\Delta\left(r_{k} \Phi\left(\Delta x_{k}\right)\right)+\left(c_{k}+\tilde{c}_{k}\right) \Phi\left(x_{k+1}\right)=0
$$

is compared with that of the linear equation

$$
\Delta\left(R_{k}^{0} \Delta y_{k}\right)+C_{k}^{0} y_{k+1}=0, \quad R_{k}^{0}:=\frac{2}{q} r_{k} h_{k} h_{k+1}\left|\Delta h_{k}\right|^{p-2}, C_{k}^{0}:=\tilde{c}_{k} h_{k+1}^{p} .
$$

In part (ii) of Theorem 2 we suppose that $p \leq 2, \tilde{r}_{k} \leq 0, \tilde{c}_{k} \geq 0$. Under these conditions, if equation (34) is oscillatory, then (33) is oscillatory by [4, Theorem 3.2] and oscillation of (3) follows then by the Sturm comparison theorem, see, e.g., [1]. However, Theorem 2, part (ii) extends [4, Theorem 3.2] in case when the perturbation $\tilde{c}_{k}$ is 'not too much positive' so that equation (33) and hence also (34) is nonoscillatory.

Similarly, if $\tilde{r}_{k}=0, \tilde{c}_{k} \geq 0$, then Theorem 3 reduces to [4, Theorem 4.2] and Theorem 4 reduces to [4, Theorem 4.1]. Theorem 5 is new also in case $\tilde{r}_{k}=0$ and it allows us to drop the condition $\lim _{k \rightarrow \infty} r_{k} h_{k} \Phi\left(\Delta h_{k}\right)=\infty$ considered in [4, Theorem 4.1] and replace it by alternative conditions. This is useful when studying perturbations of the Euler-type equation, see the next section. 
Remark 2 The conditions $\tilde{r}_{k} \leq 0, \tilde{c}_{k} \geq 0$ considered in Theorem 2(ii), Theorem 4 and Theorem 5 are used to show that $w_{k}-\left(1+\tilde{r}_{k} / r_{k}\right) w_{k}^{[h]} \geq 0$, where $w$ and $w^{[h]}$ are the solutions of the Riccati type equations associated with equations (3) and (1), respectively. We conjecture that $w_{k}$ and $\left(1+\tilde{r}_{k} / r_{k}\right) w_{k}^{[h]}$ can be compared by another argument than Lemma 3 , so the sign restriction on the perturbation terms $\tilde{r}_{k}, \tilde{c}_{k}$ can be relaxed, similarly as in the continuous case [6].

\section{Application}

In this section, we apply the previous results to the perturbed Euler-type difference equation

$$
\Delta\left(\left(1+\tilde{r}_{k}\right) \Phi\left(\Delta x_{k}\right)\right)+\left(\frac{\gamma_{p}}{(k+1)^{p}}+\bar{c}_{k}\right) \Phi\left(x_{k+1}\right)=0, \quad \gamma_{p}:=\left(\frac{p-1}{p}\right)^{p-1} .
$$

This equation is considered as a perturbation of the nonoscillatory equation

$$
\Delta\left(\Phi\left(\Delta x_{k}\right)\right)+c_{k} \Phi\left(x_{k+1}\right)=0
$$

where

$$
c_{k}=-\frac{\Delta \Phi\left(\Delta h_{k}\right)}{\Phi\left(h_{k+1}\right)}, \quad h_{k}=k^{\frac{p-1}{p}} .
$$

It is easy to see that $h_{k}=k^{\frac{p-1}{p}}$ is a solution of (36) and it was shown in [10] that if $p \geq 2$, then it is the recessive solution. By a direct computation, as shown in [4], the coefficient $c_{k}$ is of the form

$$
c_{k}=\frac{\gamma_{p}}{(k+1)^{p}}\left(1+O\left((k+1)^{-1}\right)\right), \quad \text { as } k \rightarrow \infty
$$

and also

$$
\begin{aligned}
& \Delta h_{k}=\frac{p-1}{p} k^{-\frac{1}{p}}\left(1+O\left(k^{-1}\right)\right), \\
& h_{k} \Phi\left(\Delta h_{k}\right)=\left(\frac{p-1}{p}\right)^{p-1}\left(1+O\left(k^{-1}\right)\right), \\
& h_{k} h_{k+1}\left(\Delta h_{k}\right)^{p-2}=\left(\frac{p-1}{p}\right)^{p-2} k\left(1+O\left(k^{-1}\right)\right), \\
& \Delta \Phi\left(\Delta h_{k}\right)=-\left(\frac{p-1}{p}\right)^{p}(k+1)^{-2+\frac{1}{p}}\left(1+O\left((k+1)^{-1}\right)\right),
\end{aligned}
$$

as $k \rightarrow \infty$. Consequently,

$$
\frac{\Delta h_{k}}{h_{k}}=\frac{p-1}{p} k^{-1}\left(1+O\left(k^{-1}\right)\right), \quad \text { as } k \rightarrow \infty
$$

hence conditions (13) are satisfied and we have

$$
G_{k}=\left(\frac{p-1}{p}\right)^{p-1}\left(1+\tilde{r}_{k}\right)\left(1+O\left(k^{-1}\right)\right), \quad R_{k}=2\left(\frac{p-1}{p}\right)^{p-1}\left(1+\tilde{r}_{k}\right) k\left(1+O\left(k^{-1}\right)\right),
$$


and

$$
\begin{aligned}
C_{k}= & \Delta \tilde{r}_{k} \Phi\left(\Delta h_{k+1}\right) h_{k+1}+\tilde{r}_{k} \Delta \Phi\left(\Delta h_{k}\right) h_{k+1}+\left(\bar{c}_{k}-O\left((k+1)^{-p-1}\right)\right) h_{k+1}^{p} \\
= & \left(\frac{p-1}{p}\right)^{p-1} \Delta \tilde{r}_{k}\left(1+O\left((k+1)^{-1}\right)\right)-\left(\frac{p-1}{p}\right)^{p}(k+1)^{-1} \tilde{r}_{k}\left(1+O\left((k+1)^{-1}\right)\right) \\
& +(k+1)^{p-1} \bar{c}_{k}-O\left((k+1)^{-2}\right),
\end{aligned}
$$

as $k \rightarrow \infty$. Using these computations, Corollary 1 applied to (35) reads as follows.

Corollary 2 Let $C_{k}$ be given in (38) and suppose that

$$
\sum^{\infty} C_{k}<\infty, \quad C_{k} \geq 0 \text { for large } k, \quad \liminf _{k \rightarrow \infty}\left(1+\tilde{r}_{k}\right)>0, \quad \sum^{\infty} \frac{1}{\left(1+\tilde{r}_{k}\right) k}=\infty .
$$

(i) If

$$
\limsup _{k \rightarrow \infty} \sum^{k-1} \frac{1}{\left(1+\tilde{r}_{j}\right) j} \sum_{j=k}^{\infty} C_{j}<\frac{1}{2}\left(\frac{p-1}{p}\right)^{p-1}
$$

then equation (35) is nonoscillatory.

(ii) Suppose moreover that $p \geq 2, \lim _{k \rightarrow \infty} \bar{c}_{k}(k+1)^{p+1}=\infty, \tilde{r}_{k} \leq 0$ for large $k$. If

$$
\liminf _{k \rightarrow \infty} \sum^{k-1} \frac{1}{\left(1+\tilde{r}_{j}\right) j} \sum_{j=k}^{\infty} C_{j}>\frac{1}{2}\left(\frac{p-1}{p}\right)^{p-1},
$$

then equation (35) is oscillatory.

\section{Competing interests}

The author declares that she has no competing interests.

\section{Acknowledgements}

Research supported by the Grant P201/10/1032 of the Czech Science Foundation.

Received: 23 January 2012 Accepted: 11 June 2012 Published: 5 July 2012

\section{References}

1. Řehák, P: Oscillatory properties of second order half-linear difference equations. Czechoslov. Math. J. 51, 303-321 (2001)

2. Agarwal, RP, Bohner, M, Grace, SR, O'Regan, D: Discrete Oscillation Theory. Hindawi Publishing Corporation, New York (2005)

3. Došlý, O, Řehák, P: Half-Linear Differential Equations. North-Holland Mathematics Studies, vol. 202. Elsevier, Amsterdam (2005)

4. Došlý, O, Fišnarová, S: Linearized Riccati technique and (non)oscillation criteria for half-linear difference equations. Adv. Differ. Equ. 2008, Article ID 438130 (2008)

5. Došlý, O, Rehák, P: Recessive solution of half-linear second order difference equations. J. Differ. Equ. Appl. 9, 49-61 (2003)

6. Došlý, O, Fišnarová, S: Half-linear oscillation criteria: Perturbation in term involving derivative. Nonlinear Anal., Theory Methods Appl. 73, 3756-3766 (2010)

7. Došlý, O, Fišnarová, S: Variational technique and principal solution in half-linear oscillation criteria. Appl. Math. Comput. 217, 5385-5391 (2011)

8. Došlý, O: Oscillation criteria for higher order Sturm-Liouville difference equations. J. Differ. Equ. Appl. 4, 425-450 (1998)

9. Erbe, LH, Zhang, BG: Oscillation of second order linear difference equations. Chin. J. Math. 16, 239-252 (1988)

10. Došlý, O, Fišnarová, S: Summation characterization of the recessive solution for half-linear second order difference equations. Adv. Differ. Equ. 2009, Article ID 521058 (2009) 
doi:10.1186/1687-1847-2012-101

Cite this article as: Fišnarová: Oscillatory properties of half-linear difference equations: two-term perturbations. Advances in Difference Equations 2012 2012:101.

Submit your manuscript to a SpringerOpen ${ }^{\circ}$ journal and benefit from:

- Convenient online submission

- Rigorous peer review

- Immediate publication on acceptance

- Open access: articles freely available online

- High visibility within the field

- Retaining the copyright to your article

Submit your next manuscript at $\gg$ springeropen.com 